\title{
FPS Overseas Correspondents
}

In April I967 the FPS formed a panel of consultants and correspondents to keep the Society in touch with conservation work, problems and progress overseas. This is an up-to-date list of those who are helpin this way, to whom we are most grateful.

\section{Africa}

$\begin{array}{ll}\text { Botswana } & \text { L. D. Tennant } \\ \text { Ethiopia } & \text { Melvin Bolton } \\ \text { Ghana } & \text { E. O. A. Asibey } \\ \text { Kenya } & \text { Leslie Brown } \\ & \text { Michael Sawyer } \\ \text { Malagasy Rep. } & \text { C. E. Norris } \\ \text { Malawi } & \text { Gr J. J. Petter } \\ \text { Nigeria } & \text { D. D. Hayes } \\ \text { Rhodesia } & \text { Bruce A. Happold } \\ & \text { Reay Smithers } \\ \text { Sierra Leone } & \text { Professor D. F. Owen } \\ \text { South Africa } & \text { Professor R. Bigalke } \\ & \text { Dr R. C. Bigalke } \\ & \text { Mrs C. K. Niven } \\ \text { Sudan } & \text { W. R. Siegfried } \\ \text { Tanzania } & \text { M. K. Shawki } \\ \text { The Gambia } & \text { Dr Hugh Lamprey } \\ \text { Uganda } & \text { E. F. Brewer } \\ & \text { Roger Wheater } \\ \text { Zambia } & \text { Michael Woodford, MRCvs } \\ & \text { Lieut. Colonel R. A. Critchley, Dso, MC } \\ & \text { Patrick Macartney }\end{array}$

Asia

Brunei
Burma
Ceylon
India

Indonesia
Iran
Israel
Jordan
Malaysia
Nepal
Pakistan

P. M. Shariffuddin

U. Tun Yin

Th. W. Hoffman

Dr Robin Banerjee

Zafar Futehally

Ranjit Sinh IAS

Mrs A. Wright

Hasan Basjarudin

Eskander Firouz

Dr A. Zahavi

Anis Mouasher

Professor E. Balasingam

John Blower

Syed Asad Ali

Major Ian Grimwood

Major S. Amanullah Khan

T. J. Roberts

Christopher Savage 
Sarawak

S.E. Asia
Lucas Chin

Dr Tom Harrisson

\section{Australasia}

Australia

New Guinea

Dr H. Robert Bustard Mrs Judith Wright McKinney Graham Pizzey

Harry Shugg

Angus F. Hutton

\section{Europe}

Austria

Belgium

Germany

Hungary

Ireland

Italy

Netherlands

Sweden

U.S.S.R.

North America

U.S.A.

Canada

West Indies

Bermuda

David Wingate

\section{Latin America}

General

Argentina

Brazil

Chile

Colombia

Ecuador

Peru

Venezuela

Dr H. Freundl

E. J. J. Kesteloot

Professor Dr B. Grzimek

Dr A. Keve

Fergus O'Gorman

Dr P. L. Florio

Professor A. Toschi

Dr Antoon de Vos

Dr Maarten Bijleveld

C. J. H. Maliepaard

Dr Kai Curry-Lindahl

Dr A. G. Bannikov

Dr V. G. Heptner

Roland Clement

Dr Roger Tory Peterson

Richard H. Pough

Russell E. Train

Patrick A. Hardy

J. A. Livingstone

Dr Maria Buchinger

Sr Jorge $M$. Dimitri

Dr Jose C. M. Carvalho

J. Dalgas Frisch

A. W. Johnson

Dr Carlos Lehmann

Roger Perry

Sr Pablo Rosero

Sr Felipe Benavides, OBE

W. H. Phelps 